\title{
Beneficial Effects of the RESMENA Dietary Pattern on Oxidative Stress in Patients Suffering from Metabolic Syndrome with Hyperglycemia Are Associated to Dietary TAC and Fruit Consumption
}

Rocio de la Iglesia ${ }^{1}$, Patricia Lopez-Legarrea ${ }^{1}$, Paloma Celada ${ }^{2}$, Francisco J. Sánchez-Muniz ${ }^{2}$, J. Alfredo Martinez ${ }^{1,3, *}$ and M. Angeles Zulet ${ }^{1,3}$

1 Department of Nutrition, Food Sciences and Physiology, University of Navarra, Pamplona 31008, Spain; E-Mails: rdelaiglesi@alumni.unav.es (R.I.); pllegarrea@alumni.unav.es (P.L.-L.); mazulet@unav.es (M.A.Z.)

2 Department of Nutrition and Bromatology, The Complutense University of Madrid, Madrid 28040, Spain; E-Mails: pcelada@ucm.es (P.C.); frasan@farm.ucm.es (F.J.S.-M.)

3 CIBERobn Physiopathology of Obesity and Nutrition, Centre of Biomedical Research Network, Madrid 29029, Spain

* Author to whom correspondence should be addressed; E-Mail: jalfmtz@unav.es; Tel.: +34-948-425-600 (ext. 6424); Fax: +34-948-425-740.

Received: 30 January 2013; in revised form: 8 March 2013 / Accepted: 19 March 2013 / Published: 27 March 2013

\begin{abstract}
Hyperglycemia and oxidative stress are conditions directly related to the metabolic syndrome (MetS), whose prevalence is increasing worldwide. This study aimed to evaluate the effectiveness of a new weight-loss dietary pattern on improving the oxidative stress status on patients suffering MetS with hyperglycemia. Seventy-nine volunteers were randomly assigned to two low-calorie diets $(-30 \%$ Energy): the control diet based on the American Health Association criteria and the RESMENA diet based on a different macronutrient distribution (30\% proteins, 30\% lipids, $40 \%$ carbohydrates), which was characterized by an increase of the meal frequency (seven-times/day), low glycemic load, high antioxidant capacity (TAC) and high n-3 fatty acids content. Dietary records, anthropometrical measurements, biochemical parameters and oxidative stress biomarkers were analyzed before and after the six-month-long study. The RESMENA (Metabolic Syndrome Reduction in Navarra) diet specifically reduced the android fat mass and demonstrated more effectiveness on improving general oxidative stress through a greater
\end{abstract}


decrease of oxidized LDL (oxLDL) values and protection against arylesterase depletion. Interestingly, oxLDL values were associated with dietary TAC and fruit consumption and with changes on body mass index (BMI), waist circumference, fat mass and triacilglyceride (TG) levels. In conclusion, the antioxidant properties of the RESMENA diet provide further benefits to those attributable to weight loss on patients suffering Mets with hyperglycemia.

Keywords: metabolic syndrome; hyperglycemia; oxidative stress; TAC; fruit

\section{Introduction}

The prevalence of metabolic syndrome (MetS), established as the combination of central obesity and different metabolic disturbances, such as insulin resistance, hypertension and dyslipidemia, is increasing worldwide [1,2]. Among the different metabolic abnormalities encompassing MetS, insulin resistance has been considered a common manifestation of the MetS, which leads to tissue damage and health features, involving cardiovascular diseases (CVD), atherosclerosis and hypertension [3-5]. Moreover, oxidative stress has been investigated as a potential contributor to the etiology of different pathophysiological complications, including MetS and type 2 diabetes [4,6]. Therefore, many scientific efforts are under way to detect, treat and prevent MetS, focusing on lowering the risk of type 2 diabetes and oxidative stress development [7,8]. Thus, several studies have been designed and implemented to reduce these oxidative stress-related diseases based on different lifestyle modification strategies, such as giving up smoking, increasing physical activity, controlling alcohol intake, implementing healthy sleep habits, controlling anxiety and depression, losing weight and modifying unhealthy dietary patterns [7-9]. Since it has been demonstrated that central obesity is associated with increased risks of type 2 diabetes, hypertension, CVD [10,11], oxidative stress [12] and MetS manifestations in general [11], android fat mass reduction should be a main target in order to improve MetS related diseases. Concerning nutritional strategies, most of the studies have examined the effects of single dietary factors, such as the hypotriglyceridemic effect of $n$-3 fatty acids consumption [13], the protection against oxidative damage of the dietary total antioxidant capacity (TAC) [14,15], the control of blood glucose levels of low glycemic load (GL) diets [16] or the meal frequency related appetite control [17]. However, the role of a complete dietary pattern on oxidative stress and its related diseases remains unclear [18]. Thus, it was hypothesized that the combination of all these components ( $n$-3 fatty acids, TAC, GL, meal frequency) may be effective when included in an integrated adequate dietary pattern. Therefore, in the present work, the effectiveness of a new dietary strategy involving different nutritional elements is studied in order to improve oxidative stress markers, as well as biochemical and body composition measurements on a population suffering MetS with hyperglycemia. The RESMENA-S (Metabolic Syndrome Reduction in Navarra-Spain) project [19,20]. 


\section{Results and Discussion}

\subsection{Anthropometrical, Body Composition and Blood Pressure Parameters}

After the six-month trial, both control and RESMENA dietary strategies proved to be effective on improving anthropometric, body composition and blood pressure parameters (Table 1). Both groups significantly reduced the body weight, body mass index (BMI), waist circumference, waist to hip ratio (WHR), total fat mass, lean mass, fat-free mass, systolic blood pressure (SBP) and diastolic blood pressure (DBP). However, regarding the android fat mass and related waist circumference measurement, the RESMENA diet demonstrated more benefits than the control, as volunteers of the RESMENA group presented a bigger waist circumference decrease, leading to a trend towards a marginally significance between groups $(p=0.060)$. Indeed, the RESMENA subjects were the only group that significantly reduced android fat mass values $(p<0.001)$, which resulted in significant differences between groups $(p<0.044)$. As it has been previously described, central obesity is associated with increased risks of type 2 diabetes mellitus [21], hypertension, cardiovascular diseases and MetS manifestations in general [10,11]. Moreover, only the individuals belonging to RESMENA group showed a significantly decrease in their heart rate $(p<0.001)$. Therefore, although both strategies were effective on improving general anthropometric and body composition measurements, the RESMENA diet showed additional benefits that should be taken into account in future nutritional intervention research.

Table 1. Changes in anthropometric parameters, body composition, blood pressure and activity level in both experimental groups (control and Metabolic Syndrome Reduction in Navarra (RESMENA)).

\begin{tabular}{|c|c|c|c|c|c|}
\hline & \multicolumn{2}{|c|}{ Control } & \multicolumn{2}{|c|}{ RESMENA } & \multirow{2}{*}{$\begin{array}{c}P^{\dagger} \\
\text { Difference }\end{array}$} \\
\hline & Day 0 & Day 180 & Day 0 & Day 180 & \\
\hline Weight (kg) & $103.1 \pm 2.9$ & $95.35 \pm 2.9 * * *$ & $106.0 \pm 3.2$ & $96.7 \pm 3.0 * * *$ & 0.281 \\
\hline BMI $\left(\mathrm{kg} / \mathrm{m}^{2}\right)$ & $36.4 \pm 0.7$ & $33.7 \pm 0.8 * * *$ & $37.41 \pm 0.8$ & $34.12 \pm 0.8 * * *$ & 0.206 \\
\hline Waist circumference $(\mathrm{cm})$ & $114.6 \pm 2.0$ & $107.4 \pm 2.0 * * *$ & $117.2 \pm 2.1$ & $107.1 \pm 2.0 * * *$ & 0.060 \\
\hline WHR & $1.00 \pm 0.02$ & $0.97 \pm 0.02 * * *$ & $0.99 \pm 0.02$ & $0.95 \pm 0.02 * * *$ & 0.098 \\
\hline Total fat Mass (kg) & $42.3 \pm 1.5$ & $36.4 \pm 1.6 * * *$ & $45.4 \pm 1.9$ & $37.9 \pm 1.8 * * *$ & 0.139 \\
\hline Android Fat Mass (kg) & $4.7 \pm 0.2$ & $4.3 \pm 0.3$ & $5.3 \pm 0.2$ & $4.0 \pm 0.2 * * *$ & 0.044 \\
\hline Lean mass (kg) & $58.0 \pm 2.2$ & $55.6 \pm 2.1 * * *$ & $57.1 \pm 2.1$ & $55.5 \pm 2.0 * *$ & 0.197 \\
\hline Fat-free mass (kg) & $60.9 \pm 2.3$ & $58.6 \pm 2.2 * * *$ & $60.0 \pm 2.1$ & $58.4 \pm 2.1 * *$ & 0.220 \\
\hline SBP (mmHg) & $152.9 \pm 3.3$ & $138.7 \pm 2.2 * *$ & $154.2 \pm 4.4$ & $137.1 \pm 3.1 * *$ & 0.637 \\
\hline DBP (mmHg) & $86.3 \pm 1.6$ & $79.2 \pm 1.8 * *$ & $85.8 \pm 1.8$ & $79.5 \pm 2.0 *$ & 0.766 \\
\hline Heart rate (bpm) & $75 \pm 3$ & $72 \pm 3$ & $82.3 \pm 2.6$ & $72.1 \pm 2.5 * * *$ & 0.587 \\
\hline Activity level $^{1}$ & $1.59 \pm 0.04$ & $1.54 \pm 0.04$ & $1.54 \pm 0.03$ & $1.55 \pm 0.03$ & 0.191 \\
\hline
\end{tabular}

Abbreviations: BMI, body mass index; WHR, waist to hip ratio; SBP, systolic blood pressure; DBP, diastolic blood pressure; Symbols: ** $p<0.005 ;{ }^{* * *} p<0.001$ (comparison between day 0 and day 180 in each group); $P^{\dagger}$, comparison between dietary group differences. ${ }^{1}$ Average daily exercise calculated by twenty forth physical activity questionnaire.

Regarding physical activity, as designed, volunteers of both dietary patterns maintained their activity levels along the study, with no significant differences between groups (Table 1). Therefore, the 
effects on anthropometric and biochemical parameters cannot be related to changes in physical activity, but to the different dietary patterns.

\subsection{General Biochemical Parameters}

Regarding biochemical values (Table 2), both, control and RESMENA diets, proved to be effective on ameliorating the plasma biochemical profile. As it was mentioned before, insulin resistance has been postulated as a major risk condition for the MetS development [3]. Volunteers of both groups significantly reduced their insulin and Homeostasis Model Assessment Index (HOMA-IR) values, although only those under RESMENA dietary patterns ended with significantly lower glucose levels. These results agree with the review and meta-analysis carried out by Santos et al. [22], where it was described that caloric restriction, despite the type of diet, leads to an improvement on insulin, HOMA-IR and plasma glucose levels, but the intake of a low-carbohydrate diet demonstrated a markedly bigger effect on decreasing fasting plasma glucose levels. Since volunteers included in this study presented hyperglycemia, the fact that the RESMENA group were the only that significantly decreased the glucose values has to be highlighted and might be considered in future dietary treatments of hyperglycemic patients.

Table 2. Changes in biochemical parameters in both experimental groups (control and RESMENA).

\begin{tabular}{|c|c|c|c|c|c|}
\hline & \multicolumn{2}{|c|}{ Control } & \multicolumn{2}{|c|}{ RESMENA } & \multirow{2}{*}{$\begin{array}{c}P^{\dagger} \\
\text { Difference }\end{array}$} \\
\hline & Day 0 & Day 180 & Day 0 & Day 180 & \\
\hline Total Cholesterol (mmol/L) & $5.56 \pm 0.19$ & $5.66 \pm 0.19$ & $5.44 \pm 0.21$ & $5.44 \pm 0.20$ & 0.397 \\
\hline HDL-c (mmol/L) & $1.14 \pm 0.05$ & $1.28 \pm 0.06 * * *$ & $1.11 \pm 0.04$ & $1.15 \pm 0.04$ & 0.057 \\
\hline LDL-c (mmol/L) & $3.47 \pm 0.18$ & $4.38 \pm 0.17 * * *$ & $3.34 \pm 0.17$ & $4.29 \pm 0.19 * * *$ & 0.884 \\
\hline LDL-c/ApoB & $1.43 \pm 0.04$ & $1.91 \pm 0.04 * * *$ & $1.50 \pm 0.11$ & $1.92 \pm 0.03 * *$ & 0.593 \\
\hline TG (mmol/L) & $2.06 \pm 0.21$ & $1.67 \pm 0.21 *$ & $2.17 \pm 0.21$ & $1.72 \pm 0.20 * *$ & 0.574 \\
\hline Apo A-I (mg/dL) & $134.3 \pm 4.3$ & $139.2 \pm 4.1$ & $126.3 \pm 3.5$ & $131.2 \pm 4.3$ & 0.978 \\
\hline Apo B (mg/dL) & $93.4 \pm 3.7$ & $88.7 \pm 3.4$ & $90.3 \pm 4.6$ & $86.9 \pm 4.1$ & 0.737 \\
\hline FFA $(\mathrm{mmol} / \mathrm{L})$ & $0.55 \pm 0.04$ & $0.48 \pm 0.04$ & $0.60 \pm 0.18$ & $0.50 \pm 0.23 *$ & 0.349 \\
\hline Glucose (mmol/L) & $7.14 \pm 0.36$ & $6.68 \pm 0.28$ & $7.59 \pm 0.43$ & $6.49 \pm 0.35 * *$ & 0.118 \\
\hline Insulin $(\mu \mathbf{U} / \mathbf{m L})$ & $15.22 \pm 1.56$ & $10.01 \pm 1.54 * * *$ & $15.36 \pm 1.53$ & $9.41 \pm 1.21 * * *$ & 0.685 \\
\hline HOMA-IR & $4.92 \pm 0.55$ & $3.25 \pm 0.61 * *$ & $5.24 \pm 0.56$ & $2.80 \pm 0.37 * * *$ & 0.475 \\
\hline Uric Acid (mg/dL) & $6.08 \pm 0.21$ & $6.29 \pm 0.22$ & $6.19 \pm 0.28$ & $6.23 \pm 0.22$ & 0.310 \\
\hline Total Proteins (mg/dL) & $73.01 \pm 0.94$ & $76.30 \pm 1.19 * * *$ & $71.48 \pm 0.79$ & $73.51 \pm 0.97 *$ & 0.186 \\
\hline eGFRs $\left(\mathrm{mL} / \mathrm{min} / 1.73 \mathrm{~m}^{2}\right)$ & $83.97 \pm 2.92$ & $79.85 \pm 2.60$ & $79.07 \pm 2.72$ & $81.46 \pm 3.08$ & 0.080 \\
\hline $\operatorname{ALT}(\mathbf{U} / \mathbf{L})$ & $41.59 \pm 4.29$ & $27.16 \pm 1.56 * *$ & $28.90 \pm 2.13$ & $22.54 \pm 1.60 * *$ & 0.172 \\
\hline AST (U/L) & $27.73 \pm 2.26$ & $22.86 \pm 1.15^{*}$ & $22.68 \pm 1.08$ & $20.38 \pm 1.00$ & 0.685 \\
\hline
\end{tabular}

Abbreviations: HDL-c, high density lipoprotein cholesterol; LDL-c, low density lipoprotein cholesterol; TG, triacilglycerides; Apo A-I, apolipoprotein A-I; Apo B, apolipoprotein B; FFA, free fatty acids; HOMA-IR, homeostasis model assessment of insulin resistance; eGFRs, estimated glomerular filtration rates; ALT, alanine aminotranferase; AST, aspartate aminotransferase. Symbols: ${ }^{*} p<0.05 ; * * p<0.005 ; * * * p<0.001$ (comparison between day zero and day 180 in each group); $P^{\dagger}$, comparison between dietary group differences.

Furthermore, both dietary groups significantly reduced triglyceride (TG) values, a feature that has been associated with an amelioration of coronary heart disease risks [23]. However, concerning low 
density lipoprotein-cholesterol (LDL-c), unexpectedly, the two groups increased their values, results that agree with Clifton et al. [24], who described that in some cases, LDL-c may raise despite weight loss. However, this significant increase was not observed on apolipoprotein B (Apo B) concentrations, which has been considered a better predictor of cardiovascular disease than any other lipid measurement [25]. Moreover, according to the LDL/Apo B ratio that predicts the LDL-particle size, the values being significantly raised in both groups, it indicates an increase in LDL-particle size and a lower risk of ischemic cardiac events [26,27]. With regards to high density lipoprotein-cholesterol (HDL-c) concentrations, they rose in both groups, but this increase was statistically significant only in the control group, although apolipoprotein A-I (Apo A-I), a major protein component of HDL-c [28], did not show any changes in any of the dietary groups.

Some studies associate the rise of uric acid with gout, uric acid kidney stones, diabetes and hypertension, among other diseases [29], but it also has been proposed to have a protective role and to be able to function as an antioxidant [30]. In the present study, uric acid levels slightly raised in both groups; however, no significant differences were found, neither between day zero and 180, nor between dietary groups.

Interestingly, free fatty acids (FFA), which are known to impair aortic elastic function [31], were only significantly decreased in the RESMENA group.

Concerning renal function, low levels of estimated glomerular filtration rates (eGFRs) have been positively correlated to cardiovascular disease [32]. In the present study, the control group slightly decreased these values, whereas the RESMENA group mildly increased them, leading to a trend towards significance between groups. Although decreases in protein intake has been associated to increases of eGFRs [33], our results agree with other studies where protein intake was not associated with renal function [34,35].

Transaminases, mainly alanine aminotransferase (ALT), are markers of hepatocyte injury that have shown a correlation with insulin resistance and later development of diabetes [36]. Dietary weight loss has been associated with a depletion of this liver enzyme [37] irrespective of the type of diet [38], which agrees with the present study, where both control and RESMENA group volunteers significantly decreased their ALT levels. The control group lowered aspartate aminotransferase (AST) values, as well.

\subsection{Oxidative Stress Biomarkers}

Oxidative stress, defined as an imbalance between production and degradation of reactive oxygen species, is a potential biochemical mechanism involved in the pathogenesis of MetS and diabetes [39-41]. Therefore, the study of oxidative stress-related markers on people suffering MetS and/or diabetes is important to be approached in their treatment.

High levels of plasma malondialdehyde (MDA), a biomarker of lipid peroxidation [42], have been associated with type 2 diabetes [43]. Moreover, energy-restricted dietary strategies have demonstrated to be able to decrease MDA levels [44]. At the end of the study, both dietary treatments had reduced these biomarker levels; the control group showed statistically significant changes $(p=0.007)$, and the RESMENA group showed a trend towards significance $(p=0.079)$. When comparing both groups, no statistically significant differences were found (Table 3 ). 
Table 3. Changes in oxidative stress parameters in both experimental groups (control and RESMENA).

\begin{tabular}{|c|c|c|c|c|c|}
\hline & \multicolumn{2}{|c|}{ Control } & \multicolumn{2}{|c|}{ RESMENA } & \multirow{2}{*}{$\begin{array}{c}P^{\dagger} \\
\text { Difference }\end{array}$} \\
\hline & Day 0 & Day 180 & Day 0 & Day 180 & \\
\hline MDA $(\mu \mathrm{M})$ & $0.86 \pm 0.07$ & $0.75 \pm 0.07 *$ & $0.83 \pm 0.07$ & $0.76 \pm 0.05$ & 0.449 \\
\hline MPO $(\mu \mathrm{g} / \mathrm{L})$ & $71.69 \pm 7.36$ & $65.39 \pm 7.65$ & $69.53 \pm 8.39$ & $66.48 \pm 7.42$ & 0.723 \\
\hline $\operatorname{ARE}(U / L)$ & $458 \pm 44$ & $442 \pm 43$ & $370 \pm 31$ & $361 \pm 28$ & 0.778 \\
\hline ARE:HDL-c (U/mmol) & $413.6 \pm 0.1$ & $366.8 \pm 0.1 *$ & $343.8 \pm 0.1$ & $327.1 \pm 0.1$ & 0.227 \\
\hline ARE:Apo A-I (U/mg) & $0.347 \pm 0.030$ & $0.319 \pm 0.027 *$ & $0.295 \pm 0.024$ & $0.281 \pm 0.022$ & 0.424 \\
\hline oxLDL (U/L) & $35.36 \pm 1.80$ & $36.39 \pm 2.60$ & $46.53 \pm 4.46$ & $41.03 \pm 3.22 *$ & 0.025 \\
\hline oxLDL:LDL-c (U/mmol) & $10.34 \pm 0.52$ & $8.25 \pm 0.62 * *$ & $14.88 \pm 1.80$ & $9.52 \pm 0.58 * *$ & 0.046 \\
\hline oxLDL:HDL-c (U/mmol) & $30.89 \pm 1.52$ & $28.46 \pm 1.76$ & $42.78 \pm 4.19$ & $4.19 \pm 2.64 *$ & 0.186 \\
\hline oxLDL:Apo B (U/mg) & $0.038 \pm 0.002$ & $0.043 \pm 0.004$ & $0.051 \pm 0.004$ & $0.048 \pm 0.003$ & 0.040 \\
\hline
\end{tabular}

Abbreviations: MDA, malondialdehyde; MPO, myeloperoxidase; ARE, arylesterase; HDL-c, high density lipoprotein-cholesterol; ApoA1, apolipoprotein A1; oxLDL, oxidized low density lipoprotein; LDL-c, low density lipoprotein-cholesterol; ApoB, apolipoprotein B. Symbols: * $p<0.05 ; * * p<0.005 ; * * * p 0.001$ (comparison between day zero and day 180 in each group); $P^{\dagger}$, comparison between dietary group differences.

Regarding myeloperoxidase (MPO), a leucocyte-derived enzyme that catalyzes the formation of a number of reactive oxidant species and that is known to oxidize the HDL-c [45], it has been described that energy restriction diets let to depletions on its levels [46]. In the present study, both diets slightly decreased their MPO values, but no significant differences were found, neither between day zero and day 180 in each group, nor between both dietary groups (Table 3 ).

Arylesterase (ARE) activity, one of the three functions of the paraoxonase enzyme (PON1), is associated with HDL-c and has been shown to protect LDL-c and HDL-c against oxidation [47]. In diabetic patients, PON1 ARE activity dissociates from HDL-c [48]. Studies focusing on the effect of the diet on the ARE activity are scarce, but it has been reported that flavonoids, fish oil, nori algae and pomegranate-rich based diets are positively associated with PON1 ARE activity in diabetic patients [49-52]. In the present study, volunteers of the control diet decreased ARE:HDL-c ( $p=0.006)$ and ARE:Apo A-I $(p=0.029)$ ratio values, while they remained almost unchanged in the RESMENA group. Therefore, the RESMENA diet showed a specific protection effect against ARE depletion (Table 3).

Oxidation of LDL-c is considered an important cardiovascular risk factor, since it lets to foam cell formation induction, alongside propagation of atherosclerosis [53]. Moreover, oxidized-LDL (oxLDL) has been found to be a biomarker increased in type 2 diabetic patients [54]. Our results evidenced that between both dietary patterns, RESMENA is significantly more effective on reducing oxLDL $(p=0.025)$, oxLDL:LDL-c, $(p=0.046)$ and oxLDL:Apo B $(p=0.040)$ than the control diet. Moreover, the RESMENA group was the only that significantly reduced oxLDL:HDL-c values $(p=0.025)$ (Table 3$)$. These results agree with previous studies, where an inverse relationship between high TAC dietary patterns and MetS related-oxidative stress was established [15]. Moreover, when the correlation between TAC and changes on oxLDL was studied, taking into account the entire sample, that is volunteers of both control and RESMENA groups, a significant positive relationship between oxLDL reduction and TAC values was found (Figure 1). Furthermore, the same association was 
observed when studying the relationship between oxLDL and consumed energy (kcal) from fruits (Figure 2). Finally, BMI, waist circumference, fat mass and TG value reductions are associated with decreases of oxLDL circulating concentration levels, taking again into account the entire sample (Figure 2). These results correlate with other studies, where a diet-induced weight loss resulted in significant reductions of oxLDL levels [46,55].

Figure 1. Relationship between changes on oxLDL and fruits and TAC dietary records. Abbreviations: oxLDL, oxidized low density lipoprotein; TAC, total antioxidant capacity.
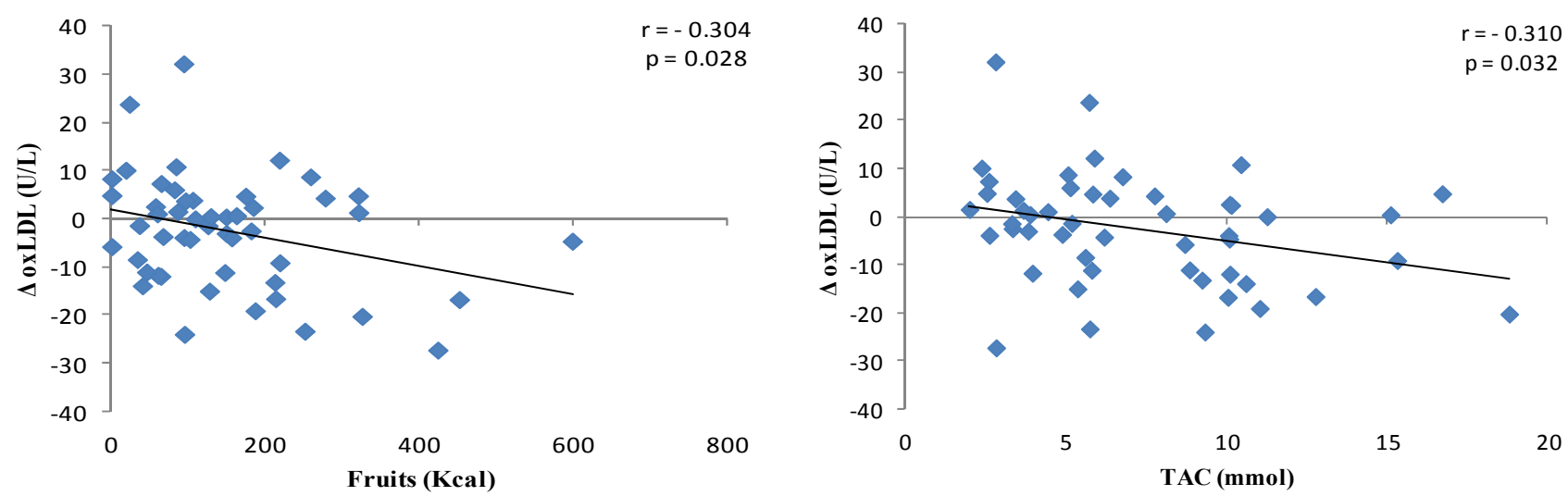

Figure 2. Correlations between changes on oxLDL and changes on adiposity parameters. Abbreviations: BMI, body mass index; TG, triglycerides.
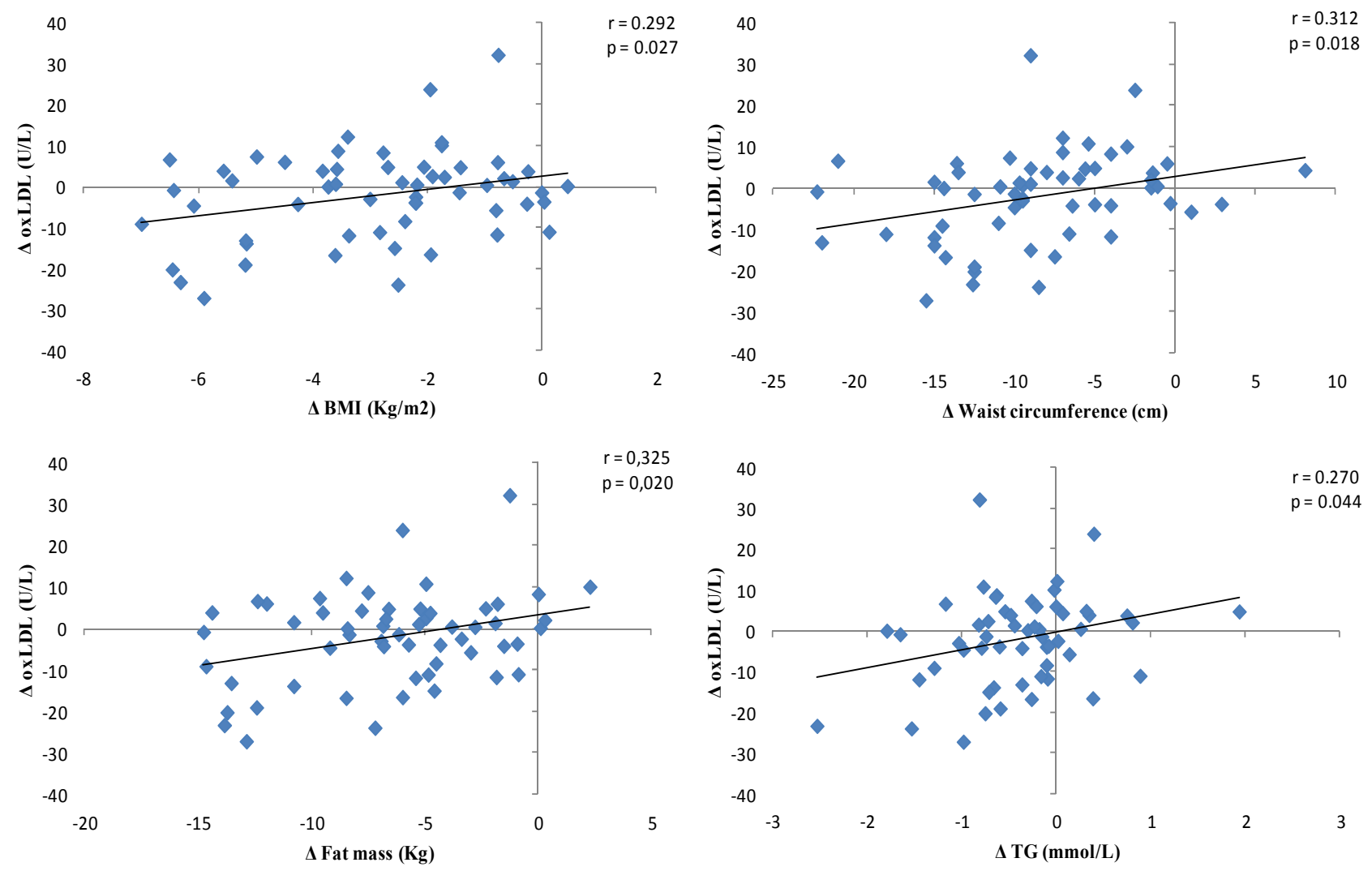


\subsection{Dietary Records}

The dietary records at the end of the study showed that the designed differences between the two dietary patterns composition were met, although no statistically significant differences were found for fiber, GL or EPA + DHA (Table 4). This outcome could be explained by the fact that the dietary records analyzed in this study were collected at the endpoint, once volunteers had completed four months of autonomy and after the six months that lasted the study. Therefore, volunteers may not complete them with the thoroughness required or might not followed the diet as strictly as at the beginning of the study. However, it was achieved that the RESMENA individuals had a higher meal frequency $(p<0.001)$, protein $(p=0.001)$ and TAC $(p=0.031)$ intake than the control group ones. Furthermore, the fruit consumption was also higher in the RESMENA group $(p=0.049)$. Moreover, both groups declared to consume the same amount of energy (Table 4), as designed. In the RESMENA group, a higher number of drop-outs than in the control group appeared, which may be a limitation of the study, although the difference was not statistically significant $(p>0.10)$.

Table 4. Comparison of control and RESMENA dietary records at the endpoint.

\begin{tabular}{cccc}
\hline & Control & RESMENA & $\boldsymbol{p}$ \\
\hline Energy (kcal/day) & $1513 \pm 54$ & $1569 \pm 77$ & 0.542 \\
Meal Frequency (meals/day) & $4.3 \pm 0.2$ & $5.8 \pm 0.2$ & $<0.001$ \\
Proteins (\% TCV/day) & $16.9 \pm 0.4$ & $20.4 \pm 0.9$ & 0.001 \\
Lipids (\% TCV/day) & $40.8 \pm 1.5$ & $37.7 \pm 1.0$ & 0.108 \\
CHO (\% TCV/day) & $37.1 \pm 1.5$ & $36.9 \pm 1.1$ & 0.940 \\
Fiber (\% TCHO/day) & $11.4 \pm 0.8$ & $12.0 \pm 0.6$ & 0.573 \\
GL (U/day) & $73.4 \pm 5.9$ & $70.0 \pm 5.5$ & 0.682 \\
EPA+DHA (g/day) & $0.30 \pm 0.08$ & $0.39 \pm 0.17$ & 0.617 \\
TAC (mmol/day) & $6.1 \pm 0.6$ & $8.5 \pm 0.9$ & 0.031 \\
Fruits (kcal/day) & $117 \pm 21$ & $185 \pm 27$ & 0.049 \\
\hline
\end{tabular}

Abbreviations: TCV, total caloric value; $\mathrm{CHO}$, carbohydrates (without fiber); TCHO, total carbohydrates (included fiber); GL, glycemic load; EPA, eicosapentaenoic acid; DHA, docosahexaenoic acid; TAC, total antioxidant capacity.

\section{Experimental Section}

\subsection{Subjects}

A subsample of 79 hyperglycemic adults diagnosed of MetS according to the IDF criteria [56] were selected from the 109 volunteers with Mets symptoms enrolled to participate in the RESMENA-S project. During the 6-month-study, 21 volunteers dropped out. Therefore, 58 individuals of the subsample completed the study and were included in the final statistical analysis (Figure 3a).

This study was conducted according to the guidelines laid down in the Declaration of Helsinki, and all procedures involving human subjects were approved by the Ethics Committee of the University of Navarra (065/2009). Written informed consent to participate in the intervention trial [20] was obtained from all subjects. 
Figure 3. Flow diagram of participants during the study (a) and ratio RESMENA/control of energy and specific dietary components of the scheduled diet (b). Abbreviations: MetS, metabolic syndrome; IDF, International Diabetes Association; MF, meal frequency; TCV, total caloric value; $\mathrm{CH}$, carbohydrates; EPA, eicosapentaenoic acid; DHA, docosahexaenoic acid; GL, glycemic load; TAC, total antioxidant capacity. Symbols: *** $p<0.001$ differences between control and RESMENA scheduled diets; N.A, not applicable.

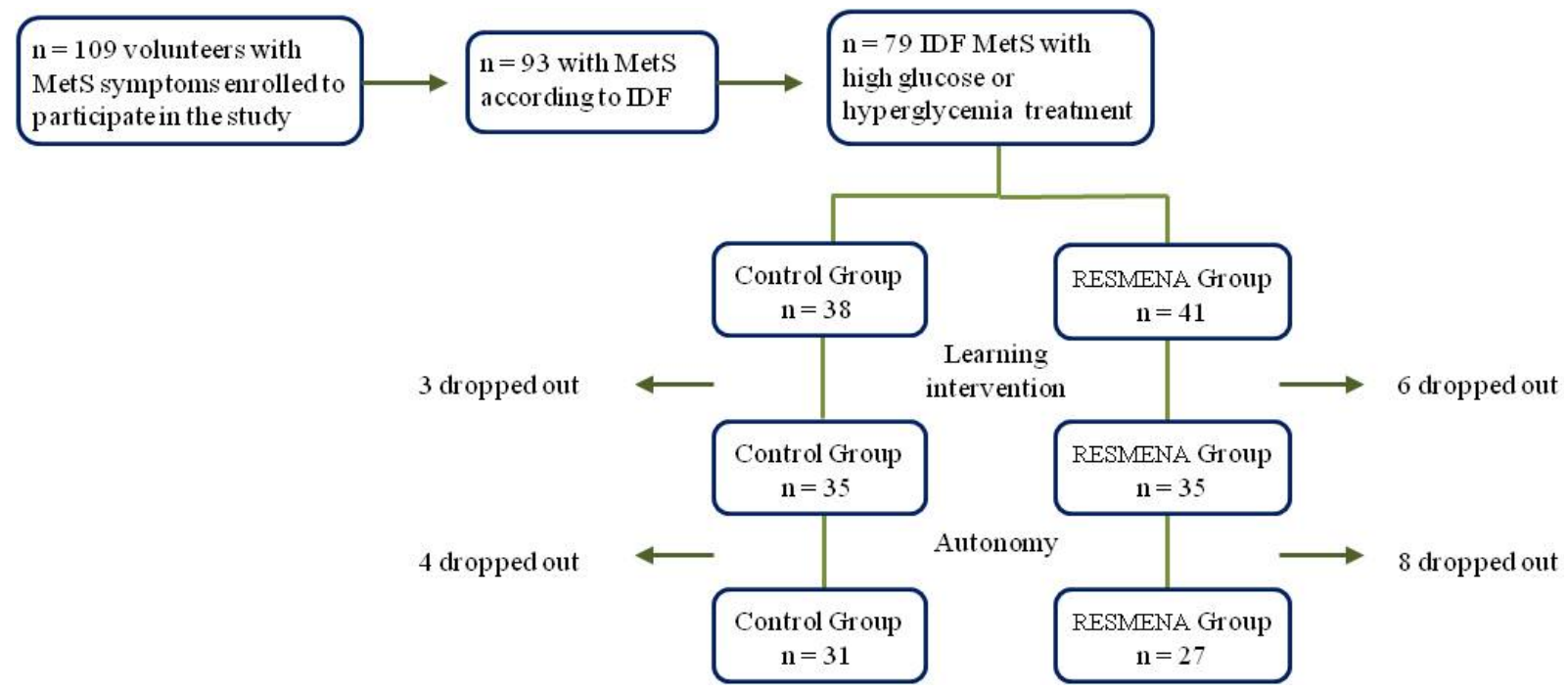

(a)

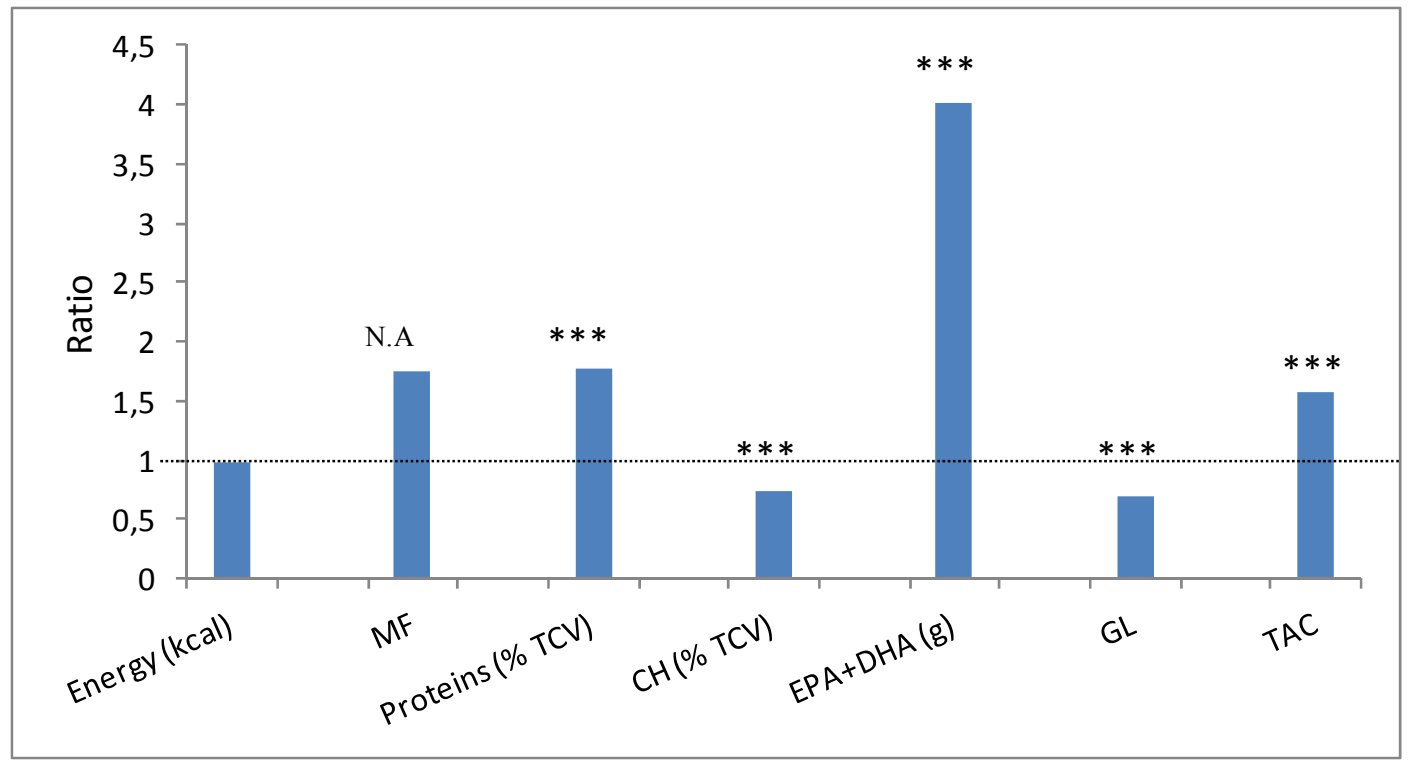

(b)

\subsection{Study Protocol}

The study was designed as a randomized, controlled trial to compare the effects of two dietary strategies (Figure 3b) on improving body composition, biochemical and oxidative stress parameters in a MetS population with hyperglycemia. Participants were randomly assigned to the control or the experimental diet (control and RESMENA groups, respectively). The study lasted a total of six months implemented in two sequential stages: an initial 8-week nutritional learning intervention period, during which the study participants received nutritional assessment every fifteen days, and a follow-up 
4-month self-control period, in which they applied on their own the previously acquired nutritional habits. The CONSORT 2010 guidelines [57] were followed by taking into account the design of the present study as two-groups longitudinal intervention, except for blinding.

Participants were asked to maintain their normal physical activity during the study, which was checked by a 24-h physical activity questionnaire [58] at the beginning and at the end of the study. For assessing physical activity, all participants were asked about their occupation, sleeping hours and additional activities at work and during the rest of the day. The physical activity questionnaire included representative values expressed as multiples of Resting Energy Expenditure. Average daily physical activity level was calculated taking into account the intensity and time spent on each activity. Activities were divided in 5 categories (resting, very light, light, moderate and heavy) [58].

At baseline and at the end point of the 6-month study, trained nutritionists performed anthropometrical measurements and body composition analyses by Dual-energy X-ray Absorptiometry (DXA) following validated protocols [19]. Moreover, fasting blood samples for biochemical analyses were collected.

\subsection{Diets}

Two energy-restricted diets $(-30 \%$ energy of the studied requirements) were prescribed and compared (Figure 3b). Thus, the control diet was based on the AHA guidelines [59], including 3-5 meals per day, a macronutrient distribution of 55\% total caloric value (TCV) from carbohydrates, $15 \%$ proteins and 30\% lipids, a healthy fatty acids (FA) profile and a cholesterol consumption lower than $300 \mathrm{mg}$ /day. The RESMENA diet was characterized by a higher meal frequency, consisting of seven meals per day and by a different macronutrient distribution, 40\% TCV from carbohydrates, 30\% proteins and 30\% lipids [19]. Furthermore, this pattern tried to reinforce the high $n$-3 polyunsaturated FA ( $n$-3 PUFAs) and high natural antioxidant foods consumption and promoted low GL carbohydrates intake. It also maintained a healthy FA profile and a cholesterol content of less than $300 \mathrm{mg} / \mathrm{day}$ as the control diet.

RESMENA participants were prescribed a 7-day menu plan, while in the control group, a previously described [60] food exchange system plan was provided to volunteers. A 48-hour weighed food record was collected at the beginning and at the end of both the nutritional-learning and the autonomous periods, in order to assess the volunteer's adherence to the prescribed nutritional patterns. The designed diets composition, as well as the different dietary records, were analyzed by the DIAL software (Alce Ingenieria, Madrid, Spain) [61]. The sum of eicosapentaenoic and docosahexaenoic fatty acid (EPA+DHA) obtained by the DIAL program [61] was used to estimate n-3 PUFAs consumption. TAC was calculated using the validated data, considering raw or cooked preparations [62]. Finally, the GL was obtained from the international updated website database based in the Human Nutrition Unit, School of Molecular Biosciences from the University of Sydney [63].

\subsection{Clinical and Biochemical Assessments}

Anthropometric measurements were performed in fasting conditions, as previously described [64]. Body weight was assessed to the nearest $0.1 \mathrm{~kg}$ by using a bioimpedance (TANITA SC-330, Tanita, Corporation, Tokyo, Japan). BMI was calculated as the body weight divided by the squared height 
$\left(\mathrm{kg} / \mathrm{m}^{2}\right)$. Waist and hip circumferences were measured with a commercial tap following validated protocols, as previously described [19]. Total body fat mass android fat mass, lean mass and fat-free mass were evaluated by DXA (Lunar iDXA ${ }^{\mathrm{TM}}$, software version 6.0, Madison, WI, USA). Measurements of SBP, DBP and heart rate were assessed using a digital monitor (Medisana, MTC, Düsseldorf, Germany) in the right arm, with the patient seated and relaxed, with an appropriate cuff for the arm size of each patient. Measurements were taken three times after a five-minute resting period, following World Health Organization (WHO) criteria [65].

Total cholesterol, HDL-c, TG, FFA, glucose, uric acid, total proteins, creatinine, ALT and AST serum concentrations were measured in an autoanalyzer Pentra C-200 (HORIBA ABX, Madrid, Spain) with specific kits. Insulin concentrations were determined by an enzyme-linked immunosorbent assay (ELISA) kit (Mercodia, Uppsala, Sweden) in a Triturus autoanalyzer (Grifols SA, Barcelona, Spain). Insulin resistance was estimated by the Homeostasis Model Assessment Index (HOMA-IR), which was calculated as stated in the following formula: HOMA-IR $=[$ glucose $(\mathrm{mmol} / \mathrm{L}) \times$ insulin $(\mu \mathrm{U} / \mathrm{mL})] / 22.5$, as described elsewhere [66]. LDL-c levels were calculated following the Friedewald formula: LDL-c $=$ Total cholesterol - HDL-c - TG/5 [67]. Apo A-I and Apo B were measured with specific kits (Tina-quant Apolipoprotein A-I ver.2 and Tina-quant Apolipoprotein B ver.2, Mannheim, Germany) using a Roche/Hitachi autoanalyzer (Mod.904 Modular, Tokio, Japan). Estimated glomerular filtration rates (eGFRs) were calculated from serum creatinine values using the equation CKD-EPI, which takes into account sex, age and race [68].

Plasma MDA was colorimetrically determined with a commercial kit $\left(B I O X Y\right.$ TECH $^{\circledR}$ LPO-586 ${ }^{\mathrm{TM}}$, Oxis Research ${ }^{\mathrm{TM}}$, Portland, OR, USA). Each sample (200 $\mu \mathrm{L}$ of serum) was mixed with $650 \mu \mathrm{L}$ of $N$-methyl-2-phenylindole in acetonitrile and $150 \mu \mathrm{L}$ of $37 \%(12 \mathrm{~N}) \mathrm{HCl}$. Tubes were capped, mixed and incubated at $45{ }^{\circ} \mathrm{C}$ for $60 \mathrm{~min}$. Samples were centrifuged at $15,000 \times g$ for $10 \mathrm{~min}$, and the supernatant was read on a spectrophotometer at $586 \mathrm{~nm}$ (Multiskan Spectrum, Thermo Electron Corporation, Vantaa, Finland). The assay included a six-point standard curve, the measurement was performed in replicate and the mean value was computed.

Plasma ox-LDL and MPO were measured using capture ELISA assay kits from Mercodia (Uppsala, Sweden). ARE activity was measured with simulated body fluid (SBF) as buffer and phenylacetate as substrate at $\mathrm{pH} 7.34-7.4$ and $37^{\circ} \mathrm{C}$, as described elsewhere [48]. Reaction rates of ARE were followed at $270 \mathrm{~nm}$ in thermostatically controlled 10-mm Lightpath quartz cuvettes using a Shimadzu UV-2401PC spectrophotometer (Tokio, Japan). The final reaction volume in the cuvettes was $2.0 \mathrm{~mL}$, and the total time was $3 \mathrm{~min}$. One unit of ARE activity is equal to $1 \mathrm{~mol}$ of phenylacetate hydrolyzed/( $\mathrm{L} \mathrm{min})$

\subsection{Statistical Analyses}

Mean values and standard errors were reported for the measured variables. Differences between the beginning and the end of the complete study were analyzed by a paired $t$-test. The analysis between both groups (RESMENA vs. Control) was performed through an independent measures $t$-test. Correlation analyses were applied to assess the potential relationships and associations, between some components of the diet and anthropometrical and biochemical parameters variation. For drop-out analysis, the $\chi^{2}$ test was applied. The SPSS 15.1 software for Windows (SPSS Inc., Chicago, USA) was used for all statistical analyses. Values of $p<0.05$ were considered as statistically significant. 


\section{Conclusions}

Both energy-restricted dietary patterns, AHA guidelines-based diet and the RESMENA diet were successful on improving anthropometrical measurements, body composition, blood pressure levels and biochemical markers on patients suffering MetS with hyperglycemia. However, the RESMENA diet showed greater benefits regarding android fat mass reduction and improvement of the general oxidative stress status, specifically oxLDL related markers. Interestingly, dietary TAC and fruit consumption were apparently the nutritional components that potentially contributed most to the oxLDL depletion. Moreover, the decrease on BMI, waist circumference, fat mass and TG levels were also directly associated with the oxLDL decrease levels. For all of this, the prescription of the RESMENA diet is a good antioxidant dietary treatment for people suffering MetS with hyperglycemia to further improve the benefits associated to weight loss.

\section{Acknowledgments}

The present work was supported by the Health Department of the Government of Navarra (48/2009) and the Línea Especial about Nutrition, Obesity and Health (University of Navarra LE/97). The support from CIBERobn and RETICS schemes is gratefully accredited. The authors thank the volunteers for taking part in this study and the physician Blanca E. Martínez de Morentín, the nurse Salomé Pérez and the technician Verónica Ciaurriz for excellent technical assistance at the Metabolic Unit of the University of Navarra. Carlos III Health Institute provided a predoctoral grant to R. de la Iglesia ( ${ }^{\circ}$ FI10/00587).

\section{Conflict of Interest}

The authors declare no conflict of interest.

\section{References}

1. Zimmet, P.; Shaw, J.; Aschner, P.; Balkau, B.; Bennett, P.; Boyko, E.; Brunzell, J.; Chan, J.; DeFronzo, R.; Després, J.; et al. A new international diabetes federation worldwide definition of the metabolic syndrome: The rationale and the results. Rev. Esp. Cardiol. 2005, 58, 1371-1376.

2. Kassi, E.; Pervanidou, P.; Kaltsas, G.; Chrousos, G. Metabolic syndrome: Definitions and controversies. BMC Med. 2011, 9, 48.

3. Crepaldi, G. Origin and Development of the Metabolic Syndrome. In The Metabolic Syndrome at the Beginning of the XXIst Century: A Genetic and Molecular Approach; Ríos, M.S., Caro, J.F., Carraro, R., Fuentes, J.A.G., Eds.; Elsevier: Amsterdam, The Netherlands, 2005; pp. 5-13.

4. Perez-Matute, P.; Zulet, M.A.; Martinez, J.A. Reactive species and diabetes: Counteracting oxidative stress to improve health. Curr. Opin. Pharmacol. 2009, 9, 771-779.

5. Huri, Z.H.; Wee, H.F. Drug related problems in type 2 diabetes patients with hypertension: A cross-sectional retrospective study. BMC Endocr. Disord. 2013, 13, 2.

6. Mansego, M.L.; Redon, J.; Martinez-Hervas, S.; Real, J.T.; Martinez, F.; Blesa, S.; Gonzalez-Albert, V.; Saez, G.T.; Carmena, R.; Chaves, F.J. Different impacts of cardiovascular risk factors on oxidative stress. Int. J. Mol. Sci. 2011, 12, 6146-6163. 
7. Mitra, A.; Dewanjee, D; Dey, B. Mechanistic studies of lifestyle interventions in type 2 diabetes. World J. Diabetes 2012, 3, 201-207.

8. Santos, A.C.; Ebrahim, S.; Barros, H. Alcohol intake, smoking, sleeping hours, physical activity and the metabolic syndrome. Prev. Med. 2007, 44, 328-334.

9. Hermsdorff, H.H.; Barbosa, K.B.; Volp, A.C.; Puchau, B.; Bressan, J.; Zulet, M.Á.; Martínez, J.A. Vitamin $\mathrm{C}$ and fibre consumption from fruits and vegetables improves oxidative stress markers in healthy young adults. Br. J. Nutr. 2012, 107, 1119-1127.

10. Hermsdorff, H.H.; Zulet, M.A.; Puchau, B.; Martínez, J.A. Central adiposity rather than total adiposity measurements are specifically involved in the inflammatory status from healthy young adults. Inflammation 2011, 34, 161-170.

11. Isomaa, B.; Almgren, P.; Tuomi, T.; Forsén, B.; Lahti, K.; Nissén, M.; Taskinen, M.R.; Groop, L. Cardiovascular morbidity and mortality associated with the metabolic syndrome. Diabetes Care 2001, 24, 683-689.

12. Nikolopoulou, A.; Kadoglou, N.P. Obesity and metabolic syndrome as related to cardiovascular disease. Expert Rev. Cardiovasc. Ther. 2012, 10, 933-939.

13. Lopez-Huertas, E. The effect of EPA and DHA on metabolic syndrome patients: A systematic review of randomised controlled trials. Br. J. Nutr. 2012, 107, S185-S194.

14. Bahadoran, Z.; Golzarand, M.; Mirmiran, P.; Shiva, N.; Azizi, F. Dietary total antioxidant capacity and the occurrence of metabolic syndrome and its components after a 3-year follow-up in adults: Tehran Lipid and Glucose Study. Nutr. Metab. 2012, 9, 70.

15. Puchau, B.; Zulet, M.A.; de Echávarri, A.G.; Hermsdorff, H.H.; Martínez, J.A. Dietary total antioxidant capacity is negatively associated with some metabolic syndrome features in healthy young adults. Nutrition 2010, 26, 534-541.

16. Mirza, N.M.; Palmer, M.G.; Sinclair, K.B.; McCarter, R.; He, J.; Ebbeling, C.B.; Ludwig, D.S.; Yanovski, J.A. Effects of a low glycemic load or a low-fat dietary intervention on body weight in obese Hispanic American children and adolescents: A randomized controlled trial. Am. J. Clin. Nutr. 2013, 97, 276-285.

17. Jääskeläinen, A.; Schwab, U.; Kolehmainen, M.; Pirkola, J.; Järvelin, M.R.; Laitinen, J. Associations of meal frequency and breakfast with obesity and metabolic syndrome traits in adolescents of Northern Finland Birth Cohort 1986. Nutr. Metab. Cardiovasc. Dis. 2012, doi:10.1016/j.numecd.2012.07.006.

18. Barbosa, K.B.; Bressan, J.; Zulet, M.A.; Martínez Hernández, J.A. Influence of dietary intake on plasma biomarkers of oxidative stress in humans. An. Sist. Sanit. Navar. 2008, 31, 259-280.

19. Zulet, M.A.; Bondia-Pons, I.; Abete, I.; de la Iglesia, R.; López-Legarrea, P.; Forga, L.; Navas-Carretero, S.; Martínez, J.A. The reduction of the metabolyc syndrome in Navarra-Spain (RESMENA-S) study: A multidisciplinary strategy based on chrononutrition and nutritional education, together with dietetic and psychological control. Nutr. Hosp. 2011, 26, 16-26.

20. ClinicalTrials.gov. U.S. National Institutes of Health. Availbale online: http://clinicaltrials.gov/show/NCT01087086 (accessed on 21 November 2011).

21. Radzeviciene, L.; Ostrauskas, R. Body mass index, waist circumference, waist-hip ratio, waist-height ratio and risk for type 2 diabetes in women: A case-control study. Public Health 2013, doi:10.1016/j.puhe.2012.12.001. 
22. Santos, F.L.; Esteves, S.S.; da Costa Pereira, A.; Yancy, W.S., Jr.; Nunes, J.P. Systematic review and meta-analysis of clinical trials of the effects of low carbohydrate diets on cardiovascular risk factors. Obes. Rev. 2012, 13, 1048-1066.

23. Carey, V.J.; Bishop, L.; Laranjo, N.; Harshfield, B.J.; Kwiat, C.; Sacks, F.M. Contribution of high plasma triglycerides and low high-density lipoprotein cholesterol to residual risk of coronary heart disease after establishment of low-density lipoprotein cholesterol control. Am. J. Cardiol. 2010, 106, 757-763.

24. Clifton, P.M.; Keogh, J. Metabolic effects of high-protein diets. Curr. Atheroscler. Rep. 2007, 9 , 472-478.

25. McQueen, M.J.; Hawken, S.; Wang, X.; Ounpuu, S.; Sniderman, A.; Probstfield, J.; Steyn, K.; Sanderson, J.E.; Hasani, M.; Volkova, E.; et al. Lipids, lipoproteins, and apolipoproteins as risk markers of myocardial infarction in 52 countries (the INTERHEART study): A case-control study. Lancet 2008, 372, 224-233.

26. Tani, S.; Saito, Y.; Anazawa, T.; Kawamata, H.; Furuya, S.; Takahashi, H.; Iida, K.; Matsumoto, M.; Washio, T.; Kumabe, N.; et al. Low-density lipoprotein cholesterol/apolipoprotein $\mathrm{B}$ ratio may be a useful index that differs in statin-treated patients with and without coronary artery disease: A case control study. Int. Heart J. 2011, 52, 343-347.

27. Sanchez-Muniz, F.J.; Bastida, S.; Viejo, J.M.; Terpstra, A.H. Small supplements of $n$-3 fatty acids change serum low density lipoprotein composition by decreasing phospholid and apolipoprotein B concentrations in young adult women. Eur. J. Nutr. 1999, 38, 20-27.

28. Miles, R.R.; Perry, W.; Haas, J.V.; Mosior, M.K.; N'cho, M.; Wang, J.W.; Yu, P.; Calley, J.; Yue, Y.; Carter, Q.; et al. Genome-wide screen for modulation of hepatic Apolipoprotein A-I (ApoA-I) secretion. J. Biol. Chem. 2013, doi:10.1074/jbc.M112.410092.

29. Johnson, R.J.; Lanaspa, M.A.; Gaucher, E.A. Uric acid: A danger signal from the RNA world that may have a role in the epidemic of obesity, metabolic syndrome, and cardiorenal disease: Evolutionary considerations. Semin. Nephrol. 2011, 31, 394-399.

30. Pasalic, D.; Marinkovic, N.; Feher-Turkovic, L. Uric acid as one of the important factors in multifactorial disorders-Facts and controversies. Biochem. Med. 2012, 22, 63-75.

31. Rider, O.J.; Holloway, C.J.; Emmanuel, Y.; Bloch, E.; Clarke, K.; Neubauer, S. Increasing plasma free fatty acids in healthy subjects induces aortic distensibility changes seen in obesity. Circ. Cardiovasc. Imaging 2012, 5, 367-375.

32. Yahalom, G.; Kivity, S.; Segev, S.; Sidi, Y.; Kurnik, D. Estimated glomerular filtration rate in a population with normal to mildly reduced renal function as predictor of cardiovascular disease. Eur. J. Prev. Cardiol. 2013, doi:10.1177/2047487313476963.

33. Wu, H.L.; Sung, J.M.; Kao, M.D.; Wang, M.C.; Tseng, C.C.; Chen, S.T. Nonprotein calorie supplement improves adherence to low-protein diet and exerts beneficial responses on renal function in chronic kidney disease. J. Ren. Nutr. 2012, doi:10.1053/j.jrn.2012.09.003.

34. Brinkworth, G.D.; Buckley, J.D.; Noakes, M.; Clifton, P.M. Renal function following long-term weight loss in individuals with abdominal obesity on a very-low-carbohydrate diet $v s$ high-carbohydrate diet. J. Am. Diet. Assoc. 2010, 110, 633-638. 
35. Beasley, J.M.; Aragaki, A.K.; LaCroix, A.Z.; Neuhouser, M.L.; Tinker, L.F.; Cauley, J.A.; Ensrud, K.E.; Jackson, R.D.; Prentice, R.L. Higher biomarker-calibrated protein intake is not associated with impaired renal function in postmenopausal women. J. Nutr. 2011, 141, 1502-1507.

36. Larson-Meyer, D.M.; Newcomer, B.R.; Heilbronn, L.K.; Volaufova, J.; Smith, S.R.; Alfonso, A.J.; Lefevre, M.; Rood, J.C.; Williamson, D.A.; Ravussin, E.; et al. Effect of 6-month calorie restriction and exercise on serum and liver lipids and markers of liver function. Obesity 2008, 16, 1355-1362.

37. Straznicky, N.E.; Lambert, E.A.; Grima, M.T.; Eikelis, N.; Nestel, P.J.; Dawood, T.; Schlaich, M.P.; Masuo, K.; Chopra, R.; Sari, C.I.; et al. The effects of dietary weight loss with or without exercise training on liver enzymes in obese metabolic syndrome subjects. Diabetes Obes. Metab. 2012, 14, 139-148.

38. Rodríguez-Hernández, H.; Cervantes-Huerta, M.; Rodríguez-Moran, M.; Guerrero-Romero, F. Decrease of aminotransferase levels in obese women is related to body weight reduction, irrespective of type of diet. Ann. Hepatol. 2011, 10, 486-492.

39. Jialal, I.; Devaraj, S.; Adams-Huet, B.; Chen, X.; Kaur, H. Increased cellular and circulating biomarkers of oxidative stress in nascent metabolic syndrome. J. Clin. Endocrinol. Metab. 2012, 97, E1844-E1850.

40. De la Iglesia, R.; Milagro, F.I.; Campión, J.; Boqué, N.; Martínez, J.A. Healthy properties of proanthocyanidins. Biofactors 2010, 36, 159-168.

41. Kaneto, H.; Matsuoka, T.A. Involvement of oxidative stress in suppression of insulin biosynthesis under diabetic conditions. Int. J. Mol. Sci. 2012, 13, 13680-13690.

42. Fernández-Sánchez, A.; Madrigal-Santillán, E.; Bautista, M.; Esquivel-Soto, J.; Morales-González, Á.; Esquivel-Chirino, C.; Durante-Montiel, I.; Sánchez-Rivera, G.; Valadez-Vega, C.; Morales-González, J.A. Inflammation, oxidative stress, and obesity. Int. J. Mol. Sci. 2011, 12, 3117-3132.

43. Kaefer, M.; de Carvalho, J.A.; Piva, S.J.; da Silva, D.B.; Becker, A.M.; Sangoi, M.B.; Almeida, T.C.; Hermes, C.L.; Coelho, A.C.; Tonello, R.; et al. Plasma malondialdehyde levels and risk factors for the development of chronic complications in type 2 diabetic patients on insulin therapy. Clin. Lab 2012, 58, 973-978.

44. Crujeiras, A.B.; Parra, D.; Goyenechea, E.; Abete, I.; González-Muniesa, P.; Martínez, J.A. Energy restriction in obese subjects impact differently two mitochondrial function markers. J. Physiol. Biochem. 2008, 64, 211-219.

45. Nicholls, S.J.; Hazen, S.L. Myeloperoxidase and cardiovascular disease. Arterioscler Thromb. Vasc. Biol. 2005, 25, 1102-1111.

46. Rector, R.S.; Warner, S.O.; Liu, Y.; Hinton, P.S.; Sun, G.Y.; Cox, R.H.; Stump, C.S.; Laughlin, M.H.; Dellsperger, K.C.; Thomas, T.R. Exercise and diet induced weight loss improves measures of oxidative stress and insulin sensitivity in adults with characteristics of the metabolic syndrome. Am. J. Physiol. Endocrinol. Metab. 2007, 293, E500-E506.

47. Nus, M.; Frances, F.; Librelotto, J.; Canales, A.; Corella, D.; Sánchez-Montero, J.M.; Sánchez-Muniz, F.J. Arylesterase activity and antioxidant status depend on PON1-Q192R and PON1-L55M polymorphisms in subjects with increased risk of cardiovascular disease consuming walnut-enriched meat. J. Nutr. 2007, 137, 1783-1788. 
48. Nus, M.; Sanchez-Muniz, F.J.; Sanchez-Montero, J.M. A new method for the determination of arylesterase activity in human serum using simulated body fluid. Atherosclerosis 2006, 188, $155-159$.

49. Sanchez-Muniz, F.J. Dietary fibre and cardiovascular health. Nutr. Hosp. 2012, 27, 31-45.

50. Lixandru, D.; Mohora, M.; Coman, A.; Stoian, I.; van Gils, C.; Aerts, P.; Manuel-Y-Keenoy, B. Diet and paraoxonase 1 enzymatic activity in diabetic foot patients from Romania and Belgium: Favorable association of high flavonoid dietary intake with arylesterase activity. Ann. Nutr. Metab. 2010, 56, 294-301.

51. Rock, W.; Rosenblat, M.; Miller-Lotan, R.; Levy, A.P.; Elias, M.; Aviram, M. Consumption of wonderful variety pomegranate juice and extract by diabetic patients increases paraoxonase 1 association with high-density lipoprotein and stimulates its catalytic activities. J. Agric. Food Chem. 2008, 56, 8704-8713.

52. Ghorbanihaghjo, A.; Kolahi, S.; Seifirad, S.; Rashtchizadeh, N.; Argani, H.; Hajialilo, M.; Khabazi, A.; Alizadeh, S.; Bahreini, E. Effect of fish oil supplements on serum paraoxonase activity in female patients with rheumatoid arthritis: A double-blind randomized controlled trial. Arch. Iran Med. 2012, 15, 549-552.

53. Hayden, M.R.; Tyagi, S.C. Homocysteine and reactive oxygen species in metabolic syndrome, type 2 diabetes mellitus, and atheroscleropathy: The pleiotropic effects of folate supplementation. Nutr. J. 2004, 10, 4.

54. Colas, R.; Pruneta-Deloche, V.; Guichardant, M.; Luquain-Costaz, C.; Cugnet-Anceau, C.; Moret, M.; Vidal, H.; Moulin, P.; Lagarde, M.; Calzada, C. Increased lipid peroxidation in LDL from type-2 diabetic patients. Lipids 2010, 45, 723-731.

55. Linna, M.S.; Borg, P.; Kukkonen-Harjula, K.; Fogelholm, M.; Nenonen, A.; Ahotupa, M.; Vasankari, T.J. Successful weight maintenance preserves lower levels of oxidized LDL achieved by weight reduction in obese men. Int. J. Obes. 2007, 31, 245-253.

56. Alberti, K.G.; Zimmet, P.; Shaw, J. The metabolic syndrome-A new worldwide definition. Lancet 2005, 366, 1059-1062.

57. Moher, D.; Hopewell, S.; Schulz, K.F.; Montori, V.; Gøtzsche, P.C.; Devereaux, P.J.; Elbourne, D.; Egger, M.; Altman, D.G. CONSORT 2010 explanation and elaboration: Updated guidelines for reporting parallel group randomised trials. Int. J. Surg. 2012, 10, 28-55.

58. Navas-Carretero, S.; Pérez-Granados, A.M.; Schoppen, S.; Sarria, B.; Carbajal, A.; Vaquero, M.P. Iron status biomarkers in iron deficient women consuming oily fish versus red meat diet. J. Physiol. Biochem. 2009, 65, 165-174.

59. Grundy, S.M.; Cleeman, J.I.; Daniels, S.R.; Donato, K.A.; Eckel, R.H.; Franklin, B.A.; Gordon, D.J.; Krauss, R.M.; Savage, P.J.; Smith, S.C., Jr; et al. Diagnosis and management of the metabolic syndrome: An American Heart Association/National Heart, Lung, and Blood Institute Scientific Statement. Circulation 2005, 112, 2735-2752.

60. Abete, I.; Parra, D.; de Morentin, B.M.; Alfredo Martinez, J. Effects of two energy-restricted diets differing in the carbohydrate/protein ratio on weight loss and oxidative changes of obese men. Int. J. Food Sci. Nutr. 2009, 60, 1-13.

61. INGENIERIA, A. DIAL Programme. Evaluation and design diets programme Available online: http://www.alceingenieria.net/nutricion.htm (accessed on 1 March 2012). 
62. Carlsen, M.H.; Halvorsen, B.L.; Holte, K.; Bøhn, S.K.; Dragland, S.; Sampson, L.; Willey, C.; Senoo, H.; Umezono, Y.; Sanada, C.; et al. The total antioxidant content of more than 3100 foods, beverages, spices, herbs and supplements used worldwide. Nutr. J. 2010, 9, 3.

63. The University of Sydney. Available online: http://www.glycemicindex.com (accessed on 5 July 2012).

64. Pérez, S.; Martínez, P.M.; de Morentín, B.; Rodríguez, M.; Martínez, J. Evaluación de la variabilidad intraindividual de la medida de composición corporal mediante bioimpedancia en voluntarias sanas y su relación con el índice de masa corporal y el pliegue tricipital. Enferm. Clín. 2005, 15, 307-314.

65. Whitworth, J.A.; Chalmers, J. World health organisation-international society of hypertension (WHO/ISH) hypertension guidelines. Clin. Exp. Hypertens 2004, 26, 747-752.

66. Aller, E.E.J.G.; Abete, I.; Astrup, A.; Martinez, J.A.; van Baak, M.A. Starches, sugars and obesity. Nutrients 2011, 3, 341-369.

67. Friedewald, W.T.; Levy, R.I.; Fredrickson, D.S. Estimation of the concentration of low-density lipoprotein cholesterol in plasma, without use of the preparative ultracentrifuge. Clin. Chem. 1972, 18, 499-502.

68. Levey, A.S.; Stevens, L.A.; Schmid, C.H.; Zhang, Y.L.; Castro, A.F., 3rd; Feldman, H.I.; Kusek, J.W.; Eggers, P.; van Lente, F.; Greene, T.; et al. A new equation to estimate glomerular filtration rate. Ann. Intern. Med. 2009, 150, 604-612.

(C) 2013 by the authors; licensee MDPI, Basel, Switzerland. This article is an open access article distributed under the terms and conditions of the Creative Commons Attribution license (http://creativecommons.org/licenses/by/3.0/). 